\title{
Observation bias correction with an ensemble Kalman filter
}

\author{
By ELANA J. FERTIG ${ }^{1 *}$, SEUNG-JONG BAEK ${ }^{2}$, BRIAN R. HUNT ${ }^{3}$, EDWARD OTT ${ }^{4}$, ISTVAN \\ SZUNYOGH${ }^{5}$, JOSÉ A. ARAVÉQUIA ${ }^{6}$, EUGENIA KALNAY ${ }^{5}, \mathrm{HONG} \mathrm{LI}^{7}$ and JUNJIE LIU ${ }^{8}$, \\ ${ }^{1}$ Oncology Biostatistics, Johns Hopkins University, Baltimore, MD 21205, USA; ${ }^{2}$ Institute for Research in Electronics \\ and Applied Physics and Department of Electrical and Computer Engineering, University of Maryland, College Park, \\ MD 20742, USA ${ }^{3}$ Institute for Physical Science and Technology and Department of Mathematics, University of \\ Maryland, College Park, MD 20742, USA; ${ }^{4}$ Institute for Research in Electronics and Applied Physics, Department of \\ Electrical and Computer Engineering and Department of Physics, University of Maryland, College Park, MD 20742, \\ USA; ${ }^{5}$ Department of Atmospheric and Ocean Science and Institute for Physical Science and Technology, University \\ of Maryland, College Park, MD 20742, USA; ${ }^{6}$ Department of Atmospheric and Ocean Science and Institute for \\ Physical Science and Technology, University of Maryland, College Park, MD 20742, USA and Center for Weather \\ Forecast and Climatic Studies, Brazilian Institute of Space Research, Cahoeira Paulista, San Paulo 12630, Brazil; \\ ${ }^{7}$ Shanghai Typhoon Institute, Shanghai, China; ${ }^{8}$ Earth and Planetary Science Department, University of California, \\ Berkeley, CA 94720, USA
}

(Manuscript received 21 April 2008; in final form 24 October 2008)

\begin{abstract}
This paper considers the use of an ensemble Kalman filter to correct satellite radiance observations for state dependent biases. Our approach is to use state-space augmentation to estimate satellite biases as part of the ensemble data assimilation procedure. We illustrate our approach by applying it to a particular ensemble scheme-the local ensemble transform Kalman filter (LETKF) — to assimilate simulated biased atmospheric infrared sounder brightness temperature observations from 15 channels on the simplified parameterizations, primitive-equation dynamics (SPEEDY) model. The scheme we present successfully reduces both the observation bias and analysis error in perfect-model simulations.
\end{abstract}

\section{Introduction}

Satellite observations provide information essential to numerical weather prediction, particularly in traditionally data poor regions like the Southern Hemisphere (e.g. Kalnay, 2003). However, satellite radiance observations are subject to relatively large biases (e.g. Eyre, 1992), which have to be accounted for in the analysis process to realize the potential of the radiance observations to improve the analyses. When assimilating radiance observations directly, many data assimilation schemes rely on nonadaptive corrections of these radiance observations (Eyre, 1992; Harris and Kelly, 2001). Non-adaptive bias correction schemes, however, have some important limitations. For example, manual intervention is required to recalibrate the bias parameters and to account for new instruments, new forward operators and biases that evolve as the instrument ages (Dee, 2005). Therefore, some

\footnotetext{
*Corresponding author. e-mail: ejfertig@jhmi.edu DOI: $10.1111 /$ j.1600-0870.2008.00378.x
}

variational data assimilation schemes correct for radiance observation biases adaptively as part of the assimilation procedure (e.g. Eyre, 1992; Derber and Wu, 1998; Harris and Kelly, 2001; Dee, 2005; Auligne et al., 2007). Typically the uncertainty in the observations is assumed to be uncorrelated to the uncertainty of the forecast model state, suggesting that adaptive bias correction should be independent of the assimilation system. However, bias also results from an amalgamation of errors in mapping the forecast state into observation space via the forward operator. As such, many adaptive schemes use 'state space augmentation' (Friedland, 1969), which appends bias parameters to the model state vector and update these parameters as part of the data assimilation process. In this paper, we develop an adaptive bias-correction scheme using state-space augmentation for ensemble-based data assimilation.

Although ensemble schemes have been found to assimilate certain radiance observations with some success (Houtekamer et al., 2005; Houtekamer and Mitchell, 2006), their ability to adaptively account for radiance biases has not been demonstrated. Baek et al. (2006), Keppenne et al. (2005) and Zupanski 
and Zupanski (2006) apply state-space augmentation to estimate low dimensional biases due to model errors with an ensemble Kalman filter. The approaches of Baek et al. (2006) and Zupanski and Zupanski (2006) also take into account the correlation between uncertainties in the model bias and uncertainties in the forecast. The observation bias correction scheme that we develop for ensemble-based schemes also incorporates correlations between uncertainties in the observation bias and forecast. We expect such correlations to be particularly relevant for correcting satellite observations, which are related to atmospheric dynamics by a complex non-linear relationship that extends over broad layers of the atmosphere (e.g. Liou, 2002).

In this paper, we use state-space augmentation for ensemblebased assimilation schemes to estimate radiance observation biases online. We apply this set-up to assimilate simulated unbiased rawinsonde observations and simulated biased radiances from the atmospheric infrared sounder (AIRS), employing, as an example, a specific ensemble Kalman filter data assimilation scheme and a specific atmospheric model. In particular, as our data assimilation scheme, we employ the local ensemble transform Kalman filter (LETKF) of Hunt et al. (2007), which is based on the localization scheme of Ott et al. (2004), whereas for our forecast model, we use the 'simplified parameterizations, primitive-equation dynamics' (SPEEDY) model of Molteni (2003). To represent the fact that the true dependence of the satellite radiance bias on the model state is unknown in reality, we generate simulated AIRS radiance observations assuming one particular 'true' functional dependence of the bias on the state and estimate the parameters of another function, often used in the operational practice, to correct the bias during the assimilation. In both cases, we use the prototype Community Radiative Transfer Model (pCRTM Han et al., 2005) as the observation operator for the AIRS radiances. We simulate biased AIRS radiances by modifying the PCRTM in a non-linear manner as suggested in Watts and McNally (2004), whereas our online bias correction estimates coefficients of a linear combination of predictive quantities proposed by Cameron (2003) and Harris et al. (2004). We will demonstrate that this bias correction scheme can significantly reduce the observation bias and the analysis root mean square (rms) error associated with the simulated AIRS observations.

In Section 2, we describe the typical forms of the bias assumed for satellite radiances, and how they are usually corrected. We also describe the LETKF data assimilation scheme and our approach to state-space augmentation bias correction with LETKF. In Section 3, we present the design of our perfect model experiments with the SPEEDY model. The results of these experiments are given in Section 4, and conclusions are provided in Section 5.

\section{Satellite radiance biases}

Satellite radiance biases arise from sources including measurement biases, observation pre-processing, representation errors and errors in the radiative transfer model, $h$, which transforms model states into radiance space. Therefore, a radiance observation, $y$, is modelled to be of the form

$y=\tilde{h}\left(\mathbf{x}^{t}, \boldsymbol{\beta}\right)+\eta$.

Here, $\mathbf{x}^{t}$ is the true state of the atmosphere, $\eta$ is the mean zero, random component of the radiance error and $\tilde{h}$ is a radiative transfer operator, modified to incorporate bias parameters $\boldsymbol{\beta}$. Because it includes the bias parameters, $\tilde{h}$ will ideally correct for the biases associated with errors in the satellite calibration or the unmodified radiative transfer model, $h$. The bias parameters, $\boldsymbol{\beta}$, differ for each channel of each instrument (Eyre, 1992; Derber and $\mathrm{Wu}, 1998$; Harris and Kelly, 2001).

Typically, the radiance bias is assumed to have two sources, scan-angle bias and air-mass bias. As the name suggests, the scan angle bias depends on the magnitude of the scan angle, whereas the air mass bias depends on the atmospheric state. These radiance biases may also have latitudinal dependence. We only consider the air-mass bias in this paper, but note that the techniques described in this paper could be applied to estimate the scan-angle bias as well.

Several operational centres, including the National Centers for Environmental Prediction (NCEP) and the European Centre for Medium-Range Weather Forecasts (ECMWF), assume that the air-mass bias is a linear combination of a small set of variables derived from the model, called 'predictors'. That is,

$\tilde{h}(\mathbf{x}, \boldsymbol{\beta})=h(\mathbf{x})+\sum_{i=1}^{N} \beta_{i} p_{i}(\mathbf{x})$,

where $\mathbf{x}$ is the model state and the radiative transfer model $h(\mathbf{x})$ is derived from physical principles and each $p_{i}$ is one of $N$ predictors (Eyre, 1992; Derber and Wu, 1998; Harris and Kelly, 2001). These bias correction schemes compute instrument dependent coefficients $\beta_{i}$ that are global constants to ensure that the predictors alone account for spatial variations in the bias for each satellite instrument and channel. This formulation assumes that the bias for a given instrument and channel is the same for the same atmospheric conditions, but it allows for rapid changes of the bias when the atmospheric conditions change rapidly. We note that it is important to limit the number of parameters $\beta_{i}$ for a given channel to avoid overfitting the parameters to the observations. The issue of overfitting is of general concern in many settings, for example, in control theory it is called 'observability' and is addressed in standard textbooks (see, for example, Ogata, 1990).

In this study, we use a subset of the predictors that was found to correct AIRS biases efficiently by Cameron (2003) and Harris et al. (2004). Specifically, we choose the following predictors:

(1) $p_{1}$ : Constant term (i.e. $p_{1}=1$ ).

(2) $p_{2}: 200-50 \mathrm{hPa}$ thickness.

(3) $p_{3}: 850-300 \mathrm{hPa}$ thickness. 
(4) $p_{4}$ : Surface skin temperature (i.e. model surface temperature).

(5) $p_{5}$ : The modelled radiance itself (i.e. $p_{5}=h(\mathbf{x})$ ).

Throughout the rest of this paper, we will refer to each predictor by its index. In the numerical experiments with the SPEEDY model presented in this paper, the thicknesses used for $p_{2}$ and $p_{3}$ are found by computing the difference between the geopotential height at the appropriate levels. The SPEEDY model obtains the surface skin temperature used for $p_{4}$ from the sea surface temperature over ocean and by modifying the land-surface temperature over land to incorporate the forecasted surface pressure (Molteni, 2003).

Based on earlier studies with the high-resolution infrared sounder (Turner, 1994; Rizzi and Matricardi, 1998), Watts and McNally (2004) propose an alternate form for to correct radiance biases from AIRS. They choose a physical modification of the radiative transfer model. Normally, the radiative transfer model is given by

$h(\mathbf{x})=\tau\left(z_{0}\right) \kappa\left(z_{0}\right) B\left(T\left(z_{0}\right)\right)+\int_{z_{0}}^{\infty} B(T(z)) \frac{\partial \tau(z)}{\partial z} \mathrm{~d} z$,

where $z$ is the physical height with $z_{0}$ at the surface, $\kappa\left(z_{0}\right)$ is the emissivity of the surface, $T(z)$ is the temperature profile associated with the state $\mathbf{x}$ at the latitude and longitude of the satellite observation and $B(T(z))$ is the Planck function. The term $\partial \tau / \partial z$ is called the 'weighting function' because it characterizes the contribution to the radiance from a given height when combined with the Planck function evaluated there. The function $\tau(z)$ represents transmittance from height $z$, that is, the portion of the radiation from height $z$ that reaches the top of the atmosphere. This transmittance function is given by

$\tau(z)=\exp \left(-\int_{z}^{\infty} \kappa\left(z^{*}\right) \rho\left(z^{*}\right) \mathrm{d} z^{*}\right)$.

Here, $\kappa(z)$ is the absorption coefficient from height $z$ and $\rho(z)$ is the density at that height. To correct for state-dependent biases, Watts and McNally (2004) incorporate a constant fractional error $\gamma$ in the specification of the absorption coefficient. That is, they make the following replacement

$\kappa \rightarrow \gamma \kappa$,

so that

$\tau(z) \rightarrow \tau(z)^{\gamma}$.

This $\gamma$ term can account for biases arising from errors in the absorption coefficient, in the density $\rho(z)$ and in the weighting function(Watts and McNally, 2004). To correct biases from other sources, they add a global constant, $\delta$, to the modified radiative transfer model. Again, $\gamma$ and $\delta$ are assumed to be global constants which depend only on the satellite and the channel. For most AIRS long-wave temperature channels, $\gamma \approx 1.05$ (Watts and McNally, 2004).
We use the bias form proposed by Watts and McNally (2004) with $\gamma=1.05$ and $\delta=0$ to simulate biased radiance observations whose systematic errors are at a physically realistic order of magnitude. We use the more standard bias correction form in eqs. (1) and (2) to estimate these biases. The goal of the present paper is not to address which functional form of the bias estimate should be used to correct real radiance biases, rather to show that we can efficiently estimate bias correction parameters with an ensemble Kalman filter.

\subsection{Online bias correction}

Bias parameters for radiance observations can be estimated online as part of the data assimilation by using state space augmentation (e.g. Derber and Wu, 1998; Dee, 2005). In data assimilation schemes using such an approach, the analysis updates an augmented state vector,

$\mathbf{z}=\left[\begin{array}{l}\mathbf{x} \\ \boldsymbol{\beta}\end{array}\right]$,

based on the observed information, where $\mathbf{x}$ is the model state and $\boldsymbol{\beta}$ is the vector of bias parameters for all satellite instruments and channels. That is, the analysis seeks the augmented state vector, $\mathbf{z}^{a}$, which best fits its background state (or 'first-guess', $\left.\mathbf{z}^{b}\right)$ and the available observations, $\mathbf{y}$.

Because the bias parameters are assumed to change much more slowly than the atmosphere, the evolution of the bias vectors is modelled by persistence, so that

$\boldsymbol{\beta}_{t}^{b}=\boldsymbol{\beta}_{t-1}^{a}$,

where $\boldsymbol{\beta}_{t-1}^{a}$ is the updated bias vector at the previous analysis time and $\boldsymbol{\beta}_{t}^{b}$ is the background bias vector at the current analysis time.

The state space augmentation approach also requires an estimate for the background error covariance of the augmented state vector, z. For simplicity, many 3D-VAR and 4D-VAR implementations assume that the error of each bias parameter is uncorrelated with the errors of the other bias parameters, with the errors of the bias parameters for other satellite channels and instruments and with the errors of the model state (Derber and Wu, 1998; Dee, 2005). We explore, in what follows, the effect of including a background error covariance for the augmented state vector learned through an ensemble-based Kalman filter scheme that we will introduce in the next section.

\subsection{Online bias correction with an ensemble Kalman filter}

As in Baek et al. (2006), our ensemble Kalman filter bias correction scheme uses an ensemble of augmented state vectors to estimate a flow-dependent background error covariance, $\mathbf{P}^{b}$. Whereas Baek et al. (2006) use state space augmentation to account for model biases in the data assimilation scheme, we 
apply this technique to update the observation operator to account for observation biases. Based on eq. (7), the modified observation operator for the augmented state vector $\mathbf{z}$ is given by $\tilde{h}(\mathbf{x}, \boldsymbol{\beta})=\tilde{h}(\mathbf{z})$. An ensemble of $k$ forecasts of these augmented state vectors are obtained from model runs starting from a set of $k$ initial conditions, where $\mathbf{z}^{b(i)}$ represents the forecast corresponding to the $i^{t h}$ ensemble member $(i=1, \ldots, k)$. Each ensemble member for the model variables and bias parameters is cycled in the data assimilation scheme. Here, forecasts of the atmospheric state portion $\mathbf{x}$ of $\mathbf{z}$ are obtained by integrating the atmospheric model, whereas forecasts of the bias vector portion $\boldsymbol{\beta}$ of $\mathbf{z}$ are obtained from eq. (8). The background error covariance can be obtained from the sample covariance of the ensemble members,

$\mathbf{P}^{b}=(k-1)^{-1} \mathbf{Z}^{b}\left(\mathbf{Z}^{b}\right)^{\top}$.

Here, $\top$ denotes the matrix transpose, and $\mathbf{Z}^{b}$ is the matrix of ensemble perturbations for the augmented state,

$\mathbf{Z}^{b}=\left[\mathbf{z}^{b(1)}-\mathbf{z}^{b}\left|\mathbf{z}^{b(2)}-\mathbf{z}^{b}\right| \ldots \mid \mathbf{z}^{b(k)}-\mathbf{z}^{b}\right]$,

where the 'background state' $\mathbf{z}^{b}$ is the ensemble mean,

$\mathbf{z}^{b}=k^{-1} \sum_{i=1}^{k} \mathbf{z}^{b(i)}$

Ensemble schemes generally use the Kalman filter equations to seek the linear combination of the ensemble members that best fits the available observations. Applying this methodology to the ensemble of augmented state vectors, ensemble schemes can simultaneously update the atmospheric state and the bias parameters. In what follows, we formulate the resulting analysis equations using LETKF (Hunt et al., 2007). We note, however, that a similar formulation could be applied to any ensemble Kalman filter scheme. We choose LETKF in this paper because, like its predecessor local ensemble Kalman filter (LEKF; Ott et al., 2004), it has been found to be an accurate and efficient data assimilation scheme for operational weather models (Szunyogh et al., 2005, 2008; Liu et al., 2008; Whitaker et al., 2008). Nonetheless, the bias correction scheme proposed here could correct observation biases in other ensemble-based data assimilation schemes (e.g. Houtekamer and Mitchell, 2001; Whitaker and Hamill, 2002).

Deferring for the moment our discussion of localization (as described in Section 2.3), the basic analysis equations of LETKF derived in Hunt et al. (2007) for augmented state vectors are

$$
\begin{aligned}
\mathbf{z}^{a} & =\mathbf{z}^{b}+\mathbf{Z}^{b} \tilde{\mathbf{P}}^{a}\left(\mathbf{Y}^{b}\right)^{\top} \mathbf{R}^{-1}\left(\mathbf{y}-\overline{\tilde{\mathbf{h}}\left(\mathbf{z}^{b(i)}\right)}\right), \\
\mathbf{P}^{a} & =\mathbf{Z}^{b} \tilde{\mathbf{P}}^{a}\left(\mathbf{Z}^{b}\right)^{\top}, \\
\tilde{\mathbf{P}}^{a} & =\left[(k-1) \mathbf{I}+\left(\mathbf{Y}^{b}\right)^{\top} \mathbf{R}^{-1} \mathbf{Y}^{b}\right]^{-1} .
\end{aligned}
$$

The observation operator $\tilde{\mathbf{h}}$ maps the background state into observation space for all the observations to be assimilated. The $j^{\text {th }}$ row of the vector $\overline{\mathbf{h}\left(\mathbf{z}^{b(i)}\right)}$ is the ensemble mean of the background ensemble in observation space for the $j^{\text {th }}$ observation. If the $j^{\text {th }}$ observation is unbiased, the $j^{\text {th }}$ row of the operator $\tilde{\mathbf{h}}$ maps the model state directly to observation space. Otherwise, we modify the corresponding row of $\tilde{\mathbf{h}}$ to incorporate the model state and bias parameters. This operator is also used to define the matrix of ensemble perturbations in observation space, $\mathbf{Y}^{b}$. Its $i^{\text {th }}$ column is given by

$\mathbf{Y}_{i}^{b}=\tilde{\mathbf{h}}\left(\mathbf{z}^{b(i)}\right)-\overline{\tilde{\mathbf{h}}\left(\mathbf{z}^{b(i)}\right)}$.

The information provided by the analysis error covariance matrix, $\mathbf{P}^{a}$, is used to generate the analysis ensemble consistent with the resulting analysis state. LETKF uses eq. (13) to determine the analysis of the augmented state for the $i^{\text {th }}$ ensemble by

$\mathbf{z}^{a(i)}=\mathbf{z}^{a}+\mathbf{Z}_{i}^{a}$,

where $\mathbf{Z}_{i}^{a}$ is the $i^{\text {th }}$ column of $\mathbf{Z}^{a}=(k-1)^{-1 / 2} \mathbf{Z}^{b}\left(\tilde{\mathbf{P}}^{a}\right)^{1 / 2}$. We note that LETKF, like many ensemble-based schemes, directly solves the set of analysis equations to obtain the analysis, without the 'inner-loop' iterations performed by variational schemes.

\subsection{Local analysis and global bias estimation}

Computational restrictions limit the number of ensemble members that can be used to obtain the analysis in eqs. (12)-(16). In practice, the resulting ensemble is not large enough to capture the global dynamics. However, within local regions, a limited number of ensemble members is presumed sufficient to capture the dynamics (Patil et al., 2001; Ott et al., 2004; Oczkowski et al., 2005; Szunyogh et al., 2005; Kalnay, 2006; Kuhl et al., 2007). Therefore, many ensemble schemes, including LETKF, use localization (Anderson, 2001; Hamill et al., 2001; Houtekamer and Mitchell, 2001; Whitaker and Hamill, 2002; Ott et al., 2004; Hunt et al., 2007).

As in the LEKF (Ott et al., 2004), the analysis for each gridpoint in LETKF is obtained by solving eqs. (12)-(16) exactly, using only those observations in a local region centred at that gridpoint (Ott et al., 2004; Hunt et al., 2007). Here, these regions contain $2 l+1$ gridpoints in the two horizontal directions and $2 v+1$ gridpoints in the vertical direction, for some choice of $l$ and $v$. This type of localization (Ott et al., 2004) allows fast parallel implementation (Szunyogh et al., 2005, 2008). Because satellite observations depend on deep layers of the atmosphere, they cannot be selected for assimilation in the local regions this way. Based on Fertig et al. (2007), we use a cut-off-based localization for the satellite observations. Specifically, we use a satellite observation in the analysis at a gridpoint if its weighting function is above a cut-off value, $\chi$, somewhere within the local region around that gridpoint. 
In what follows, we provide a more formal description of the localization in LETKF. Let $\mathbf{A}$ be the operator that computes an analysis ensemble $\left\{\mathbf{z}^{a(i)}\right\}$ according to eqs. (12)-(16):

$\left\{\mathbf{z}^{a(i)}\right\}=\mathbf{A}\left(\left\{\mathbf{z}^{b(i)}\right\}, \mathbf{y}\right)$.

Next, let $\mathbf{A}_{x}$ and $\mathbf{A}_{\beta}$ be the components of $\mathbf{A}$ that output the model variables $\left\{\mathbf{x}^{a(i)}\right\}$ and bias variables $\left\{\boldsymbol{\beta}^{a(i)}\right\}$, respectively. We can then rewrite eq. (17) as

$\left\{\mathbf{x}^{a(i)}\right\}=\mathbf{A}_{x}\left(\left\{\mathbf{z}^{b(i)}\right\}, \mathbf{y}\right)$,

$\left\{\boldsymbol{\beta}^{a(i)}\right\}=\mathbf{A}_{\beta}\left(\left\{\mathbf{z}^{b(i)}\right\}, \mathbf{y}\right)$.

In LETKF, the analysis is obtained independently for each gridpoint using only those observations that are thought to provide useful information about the atmospheric state at the given gridpoint. For a given gridpoint $j$, let $\mathbf{y}_{j}$ denote the local set of observations chosen for that gridpoint's analysis and let $\mathbf{A}_{x_{j}}$ denote the component of $\mathbf{A}_{x}$ that outputs only the model variables $\left\{\mathbf{x}_{j}^{a(i)}\right\}$ at gridpoint $j$. Then, the LETKF analysis for those model variables is

$\left\{\mathbf{x}_{j}^{a(i)}\right\}=\mathbf{A}_{x_{j}}\left(\left\{\mathbf{z}^{b(i)}\right\}, \mathbf{y}_{j}\right)$.

Here, $\tilde{\mathbf{h}}$ and $\mathbf{R}$ are truncated to match $\mathbf{y}_{j}$. It is important to note that this truncation is only performed after the observation operator is applied to the global background state. Thus, while using spatial localization, this scheme can nonetheless account for the full atmospheric dynamics upon which the satellite observation depends (Fertig et al., 2007).

Whereas the LETKF analysis for the model variables at each gridpoint only depends on the observations near that gridpoint, the estimate for the observation bias parameters should ideally depend on the global set of observations $\mathbf{y}$. Such global dependence would reflect the fact that observation biases arise from instrument-dependent errors, to which observations made at any location would be subject. Ideally, the predictors account for any local state dependence of these observation biases. Therefore, we assume that the bias parameters are global constants that depend on the instrument, channel, and ensemble member. For this purpose, we could simply use the global analysis in eq. (19) to produce the bias coordinates of the analysis ensemble. However, a global analysis is much more computationally expensive than a local analysis and is consequently typically infeasible.

In this paper, we propose two approaches that provide global estimates of the observation bias parameters using the local framework of the LETKF scheme. In the first, which we call the 'one-step analysis scheme', we estimate global observation bias parameters in the local analysis at each gridpoint and then perform a suitable spatial average of these estimates to obtain a single global estimate of each observation bias parameter. Although this scheme can incorporate correlations between errors in the model state and bias parameters, as we describe subsequently, it may be subject to local overfitting by the bias parameters. We, therefore, propose an alternative, 'two-step analysis scheme'. This scheme first estimates the global observation bias parameters using the one-step scheme and then updates the model state variables directly with LETKF, replacing the observation operator in the LETKF equations with an observation operator corrected by the ensemble mean of the previously estimated global observation bias parameters. We note that we must select parameters whose values vary independently over the local regions to avoid overfitting a particular parameter.

2.3.1. One-step analysis scheme In the one-step scheme, we use an approximation to eq. (19). We start with the estimates of the bias parameters produced by the local analyses

$\left\{\boldsymbol{\beta}_{j}^{a(i)}\right\}=\mathbf{A}_{\beta}\left(\left\{\mathbf{z}^{b(i)}\right\}, \mathbf{y}_{j}\right)$.

Note that the bias parameters in the background ensemble are global constants, but that each local region suggests a different correction to the bias parameters. Then, to obtain each scalar global bias parameter $\beta^{a(i)}$, we average the local estimates $\beta_{j}^{a(i)}$ over all gridpoints as follows,

$\beta^{a(i)}=\frac{\sum_{j} \cos \left(\phi_{j}\right) \beta_{j}^{a(i)} \sigma_{j}^{-2}}{\sum_{j} \cos \left(\phi_{j}\right) \sigma_{j}^{-2}}$,

Here $\phi_{j}$ is the latitude at the central gridpoint of local region $j$, which we incorporate to represent the surface area covered in each local region. We also account for the relative accuracy of the parameter estimated in each local region by incorporating in eq. (22) the local variance $\sigma_{j}^{2}$ of the analysis bias, where $\sigma_{j}^{2}$ represents this variance in the $j^{\text {th }}$ local region and is given by

$\sigma_{j}^{2}=(k-1)^{-1} \sum_{l=1}^{k}\left(\beta_{j}^{a(l)}-\beta_{j}^{a}\right)^{2}$,

where $\beta_{j}^{a}$ is the ensemble mean of the bias analysis in the $j^{\text {th }}$ local region. ${ }^{1}$

Specifically, we employ the following steps to estimate the model state variables and observation bias parameters in the one-step analysis scheme:

(1) For each ensemble member $i$, compute $\tilde{\mathbf{h}}\left(\mathbf{z}^{b(i)}\right)$ with the global constant bias parameter for that ensemble member estimated at the previous time step.

(2) For each gridpoint $j$, obtain local estimates of the model state variables and bias parameters as follows:

(a) $\left\{\mathbf{x}_{j}^{a(i)}\right\}=\mathbf{A}_{x_{j}}\left(\left\{\mathbf{z}^{b(i)}\right\}, \mathbf{y}_{j}\right)$.

(b) $\left\{\boldsymbol{\beta}_{j}^{a(i)}\right\}=\mathbf{A}_{\beta}\left(\left\{\mathbf{z}^{b(i)}\right\}, \mathbf{y}_{j}\right)$.

(3) Apply eq. (22) to obtain a global estimate for the bias parameters for each ensemble member, $\boldsymbol{\beta}^{a(i)}$.

\footnotetext{
${ }^{1}$ In the numerical experiments in this paper, we do not observe a significant difference in the analysis using simpler means of weighting the average (e.g. by the number of observations in each local region, results not shown).
} 
The one-step analysis scheme can incorporate correlations between model state variables and bias parameters by updating their states simultaneously in the local analysis phase. However, a potential concern is that by initially giving the bias parameters freedom to adjust 'locally', we may degrade the analysis obtained for the model state variables in the following sense. If the background covariance of the bias parameters is not sufficiently constrained by past analysis cycles, then the local observation increments may be fit more than optimally by local adjustments to the bias parameters and less than optimally by the model state variables. Therefore, we propose an alternative two-step analysis scheme that first updates the bias parameters globally and then computes local analyses for the model state variables with the bias parameters fixed.

2.3.2. Two-step analysis scheme The two-step analysis scheme updates the bias parameters using the methodology proposed in the one-step analysis scheme, without updating the model state variables. It then updates the model state variables with the original formulation of LETKF, applying the global and ensemble average of the bias parameters estimated in the first step to correct the observation operator. Specifically, the two-step analysis scheme follows the steps outlined below to estimate the model state variables and bias parameters:

(1) For each ensemble member $i$, compute $\tilde{\mathbf{h}}\left(\mathbf{z}^{b(i)}\right)$ with the global constant bias parameter for that ensemble member estimated at the previous time step.

(2) For each gridpoint $j$, compute $\left\{\boldsymbol{\beta}_{j}^{a(i)}\right\}=\mathbf{A}_{\beta}\left(\left\{\boldsymbol{z}^{b(i)}\right\}, \mathbf{y}_{j}\right)$.

(3) Apply eq. (22) to obtain a global estimate for the bias parameters for each ensemble member, $\boldsymbol{\beta}^{a(i)}$.

(4) For each ensemble member $i$, incorporate the bias parameters estimated in the first step by:

(a) letting $\tilde{\mathbf{z}}^{b(i)}$ represent the augmented state vector containing $\mathbf{x}^{b(i)}$ and $\overline{\boldsymbol{\beta}^{a(i)}}$, where - represents the ensemble average and

(2) computing $\tilde{\mathbf{h}}\left(\tilde{\mathbf{z}}^{b(i)}\right)$ with the global constant bias parameter averaged over all ensemble members.

(i) For each gridpoint $j$, compute $\left\{\boldsymbol{x}_{j}^{a(i)}\right\}=\mathbf{A}_{x}\left(\left\{\tilde{\mathbf{z}}^{b(i)}\right\}, \mathbf{y}_{j}\right)$.

Although the two-step analysis scheme disregards correlations between the model state variables and bias parameters, it provides independent estimates of the state and the bias, thus eliminating the potential problem in the one-step analysis scheme of local overfitting by the bias parameters.

Because both the one- and two-step analysis schemes estimate only a few bias parameters that are global constants, the dimensionality of the augmented system is not greatly increased by including the bias parameters in either analysis scheme. Therefore, regardless of the scheme that we employ, we do not require many additional ensemble members to estimate the observation biases. This is in contrast to Baek et al. (2006) which obtained a 'pointwise' estimate of the model bias. Whereas our correction to the observation bias is global, this pointwise estimate of model biases requires them to be treated locally like the model state variables. Thus, in Baek et al. (2006), a relatively large increase in the ensemble is required.

\section{Experimental design}

\subsection{SPEEDY model}

The numerical experiments in this paper are performed using the SPEEDY model (Molteni, 2003), which is a global circulation model based on the primitive equations. Due to its low resolution (T30) and simplified parametrization schemes, the computation cost of integrating the SPEEDY model is much lower than that of an operational global circulation model. The simplified physics includes the parametrization of the following processes: convection; large-scale condensation; cloud physics; radiation; surface fluxes and vertical diffusion.

The SPEEDY model obtains its forecasts by solving the primitive equations for vorticity, divergence, absolute temperature, specific humidity and the logarithm of the surface pressure at seven sigma levels $(0.950,0.835,0.685,0.510,0.340,0.200$, 0.080 ). The resulting values are converted to output variables of zonal wind, $u$, meridional wind, $v$, absolute temperature, $T$, and specific humidity, $q$ at 96 zonal gridpoints, 48 meridional gridpoints and seven sigma levels or seven pressure levels (925, $850,700,500,300,200,100 \mathrm{hPa})$. The surface pressure, $p_{s}$ is available at the same horizontal resolution but for only a single vertical level that represents the surface.

\subsection{Community radiative transfer model}

In this paper, we use the pCRTM (Han et al., 2005) to generate the simulated satellite observations and for the corresponding observation operator. This model was developed by the Joint Center for Satellite Data Assimilation (JCSDA) to provide the community with a unified code to simulate satellite radiances. As part of this mission, the framework of the pCRTM and its more advanced counterpart, the CRTM, are both general enough to be easily adapted to simulate radiances from most satellite instruments and from most atmospheric model states. Furthermore, the more advanced CRTM is currently used by the operational NCEP Global Forecast System (GFS) and the Gridpoint Statistical Interpolation System (GSI) assimilation schemes. In this paper, we use the pCRTM to simulate satellite radiance due to the relative simplicity of its software structure and underlying physics (Han et al., 2005).

The pCRTM determines the radiance and brightness temperature from an input profile of pressure, temperature, water vapour and ozone. Here, the profile values for pressure, temperature and water vapour are obtained from the SPEEDY model. For simplicity, in this study we assume that the ozone profile has a constant value of 0.06720 ppmv. Furthermore, the numerical experiments in this paper are not concerned with scan angle bias since we assume that the satellite observes the atmosphere 
directly below the location of the satellite. Moreover, the clearsky assumption made in the pCRTM (and relaxed in the CRTM) is sufficient for this study.

The pCRTM determines the radiance by solving a discrete form of eq. (3). This equation is given by

$h(\mathbf{x})=R_{\text {surface }}+\sum_{i=1}^{\text {top }} B\left(T_{i}\right) \Delta \tau_{i}$,

where $R_{\text {surface }}$ is the contribution to the radiance from the surface and $T_{i}$ is the temperature at model level $i$. The values of $\tau$ at each level of the profile are derived from information about the spectral and transmittance coefficients for the desired satellite observation. $\Delta \tau_{i}$ is the difference between the $\tau$ values at levels $i$ and $i+1$ in the profile for all levels below the top of the profile. In this study, we assume that there is no transmittance from the surface to the top of the atmosphere. As a result, $\Delta \tau$ is determined at the top of the profile by enforcing $\sum_{i=1}^{\text {top }} \Delta \tau_{i}=1$. The brightness temperature found by the pCRTM is the temperature that a black body would have a radiance equivalent to the value of the satellite radiance.

LETKF obtains the analysis independently for each gridpoint by using only those observations that are thought to provide potentially useful information about the atmospheric state at the given grid point. In practice, observations are typically chosen from a local volume centred at the given gridpoint (e.g. Szunyogh et al., 2008). When using this localization, we found that radiance observations enhance the accuracy of the analysis most when we select those observations whose weighting function such observations are assimilated at all model levels where the weighting function associated with them exceeds an empirically determined cut-off value $\chi$ (Fertig et al., 2007). In the discrete form of the radiative transfer model (eq. 24), $\Delta \tau_{i}$ indicates the influence of the radiance from the $i^{\text {th }}$ layer of the profile. Therefore, we use this value of $\Delta \tau_{i}$ to select observations using the cut-off based selection. We ran several experiments with varying values of $\chi$ and found that selecting observations with $\Delta \tau_{i}$ of at least

$\chi=0.4 \times\left(\max _{i=1, \ldots, \text { top }} \Delta \tau_{i}\right)$,

somewhere within the local region provides the smallest analysis rms error (results not shown). Accordingly, we use this value in all the numerical experiments in this paper.

\subsection{Truth and observations}

We perform the numerical experiments in the perfect model scenario with the SPEEDY model. Following Miyoshi (2005), the 'true' state of the atmosphere is obtained by running the SPEEDY model for a year from its default rest state on 1 January 1981 . We then obtain the true atmospheric trajectory by integrating the SPEEDY model from the resulting atmospheric state at 1 January 1982 for 240 time steps (roughly two months).
The quality of the analyses is assessed by comparing the resulting analysis with this true state. Specifically, we compute the global rms error between the true and analysis states in pressure coordinates. The average rms error is the average of these errors over the last month of the assimilation cycle.

We create two sets of simulated observations from the known 'true' state of the atmosphere: a set of rawinsonde observations and a set of radiance observations. The simulated rawinsonde observations are created by adding zero mean, Gaussian noise to the true state of the atmosphere every $6 \mathrm{~h}$ at those points of the SPEEDY model that are closest to the real rawinsonde observations at the $12 \mathrm{Z}$ synoptic time (Miyoshi, 2005). We use a standard deviation of $1 \mathrm{~m} \mathrm{~s}^{-1}$ for $u$ and $v, 1 \mathrm{~K}$ for $T, 0.0001$ $\mathrm{kg} \mathrm{kg}^{-1}$ for $q$ and $1 \mathrm{hPa}$ for $p_{s}$.

Similarly, we simulate 15 long-wave temperature radiance channels from AIRS 281 subset (wavenumbers: 680.491, 691.391, 694.4, 696.052, 697.99, 699.102, 700.218, 701.618, 703.87, 706.991, 709.566, 712.739, 715.941, 723.029, 724.824 $\mathrm{cm}^{-1}$ ) at each horizontal model gridpoint. These channels represent the dynamics over model levels considered by the SPEEDY model with some redundancy, to reflect the relative vertical density of satellite observations on this coarsely resolved atmospheric model (see the values of their weighting function in Table 1). The channels were selected from a subset of the long-wave temperature in the 281 subset of AIRS channels for numerical weather prediction (e.g. Le Marshall et al., 2006), which represent the independent information measured all the AIRS channels (Susskind et al., 2003). We chose only observations from temperature channels to avoid the complications that arise in assimilating water vapour observations.

These radiance observations are generated by applying the pCRTM to obtain brightness temperature observations from the true atmospheric state. A zero mean, Gaussian noise with standard deviation $0.5 \mathrm{~K}$ is added to the resulting brightness temperature observation. We selected this observation noise to be smaller than that of the rawinsondes, but large enough to be significant relative to the bias. The bias in the brightness temperature observations is simulated by modifying $\kappa(z)$ in the pCRTM as suggested by Watts and McNally (2004) and described in Section 2. Throughout this paper, we refer to the PCRTM with the modification proposed by Watts and McNally (2004) as the modified pCRTM. Because the bias is presumed unknown by the analysis scheme, the unmodified PCRTM is used for the observation operator during the data assimilation.

However, for our simulated radiances, the true bias in the observations can be calculated by applying both the modified pCRTM and unmodified pCRTM to the true state. The difference between these two values of the brightness temperature represents the true satellite bias. The extent to which the estimated bias parameters reduce the true bias provides an additional means to verify the data assimilation scheme. Furthermore, the correlation between the true bias and the predictors indicates which predictors can be expected to make the largest 
Table 1. The value of the weighting function and percent of maximum (in parentheses) on 06UTC 1 January 1982 at $180^{\circ} \mathrm{W}, 35^{\circ} \mathrm{N}$ and each model level; though at a specific date and time, these values are representative of the general values of the weighting function. Note that because the radiative transfer model numerically integrates over all model levels, the value of the weighting function is available at the midpoint between each model layer defined by two consecutive model levels. Bolded values are above the cut-off value of $\chi=0.4 \times\left(\max _{i=1, \ldots, \text { top }} \Delta \tau_{i}\right)$

\begin{tabular}{lcccccc}
\hline \multirow{2}{*}{$\begin{array}{l}\text { Channel wavenumber } \\
\left(\mathrm{cm}^{-\mathbf{1}}\right)\end{array}$} & \multicolumn{5}{c}{ Vertical level $(\mathrm{hPa})$} \\
\cline { 2 - 6 } & 0.9500 & 0.7600 & 0.5975 & 0.4250 & 0.2700 & 0.1400 \\
\hline 680.491 & $0.00(0.00)$ & $0.00(0.00)$ & $0.00(0.00)$ & $0.01(0.02)$ & $\mathbf{0 . 5 8}(\mathbf{1 . 0 0})$ & $\mathbf{0 . 4 0}(\mathbf{0 . 6 9})$ \\
691.391 & $0.00(0.00)$ & $0.00(0.00)$ & $0.00(0.00)$ & $0.00(0.00)$ & $0.06(0.06)$ & $\mathbf{0 . 9 4}(\mathbf{1 . 0 0})$ \\
694.400 & $0.00(0.00)$ & $0.00(0.00)$ & $0.00(0.00)$ & $0.02(0.02)$ & $0.13(0.15)$ & $\mathbf{0 . 8 6}(\mathbf{1 . 0 0})$ \\
696.052 & $0.00(0.00)$ & $0.00(0.00)$ & $0.00(0.00)$ & $0.03(0.04)$ & $0.17(0.21)$ & $\mathbf{0 . 8 0}(\mathbf{1 . 0 0})$ \\
697.990 & $0.00(0.00)$ & $0.00(0.00)$ & $0.00(0.00)$ & $0.01(0.01)$ & $0.13(0.15)$ & $\mathbf{0 . 8 6}(\mathbf{1 . 0 0})$ \\
699.102 & $0.00(0.00)$ & $0.00(0.00)$ & $0.00(0.00)$ & $0.02(0.02)$ & $0.05(0.05)$ & $\mathbf{0 . 9 3}(\mathbf{1 . 0 0})$ \\
700.218 & $0.00(0.00)$ & $0.00(0.00)$ & $0.00(0.00)$ & $0.00(0.00)$ & $0.00(0.00)$ & $\mathbf{1 . 0 0}(\mathbf{1 . 0 0})$ \\
701.618 & $0.00(0.00)$ & $0.00(0.00)$ & $0.00(0.00)$ & $0.00(0.00)$ & $0.00(0.00)$ & $\mathbf{1 . 0 0}(\mathbf{1 . 0 0})$ \\
703.870 & $0.00(0.00)$ & $0.00(0.00)$ & $0.02(0.03)$ & $0.09(0.13)$ & $0.19(0.27)$ & $\mathbf{0 . 7 0}(\mathbf{1 . 0 0})$ \\
706.991 & $0.00(0.00)$ & $0.01(0.02)$ & $0.05(0.10)$ & $0.17(0.34)$ & $\mathbf{0 . 2 4}(\mathbf{0 . 4 7})$ & $\mathbf{0 . 5 2}(\mathbf{1 . 0 0})$ \\
709.566 & $0.00(0.00)$ & $0.02(0.03)$ & $0.06(0.09)$ & $0.12(0.19)$ & $0.14(0.22)$ & $\mathbf{0 . 6 5}(\mathbf{1 . 0 0})$ \\
712.739 & $0.04(0.13)$ & $0.09(0.27)$ & $\mathbf{0 . 1 5}(\mathbf{0 . 4 8})$ & $\mathbf{0 . 2 0}(\mathbf{0 . 6 3})$ & $\mathbf{0 . 1 6}(\mathbf{0 . 5 3})$ & $\mathbf{0 . 3 1}(\mathbf{1 . 0 0})$ \\
715.941 & $\mathbf{0 . 0 8}(\mathbf{0 . 4 3})$ & $\mathbf{0 . 1 3}(\mathbf{0 . 7 0})$ & $\mathbf{0 . 1 8}(\mathbf{0 . 9 6})$ & $\mathbf{0 . 1 9}(\mathbf{1 . 0 0})$ & $\mathbf{0 . 1 3}(\mathbf{0 . 6 7})$ & $\mathbf{0 . 1 3}(\mathbf{0 . 6 6})$ \\
723.029 & $\mathbf{0 . 0 9}(\mathbf{0 . 5 7})$ & $\mathbf{0 . 1 2}(\mathbf{0 . 7 9})$ & $\mathbf{0 . 1 4}(\mathbf{0 . 9 6})$ & $\mathbf{0 . 1 5}(\mathbf{1 . 0 0})$ & $\mathbf{0 . 1 1}(\mathbf{0 . 7 0})$ & $\mathbf{0 . 1 3}(\mathbf{0 . 8 8})$ \\
724.824 & $\mathbf{0 . 0 9}(\mathbf{0 . 6 1})$ & $\mathbf{0 . 1 3}(\mathbf{0 . 8 4})$ & $\mathbf{0 . 1 5}(\mathbf{1 . 0 0})$ & $\mathbf{0 . 1 4}(\mathbf{0 . 9 5})$ & $\mathbf{0 . 0 9}(\mathbf{0 . 6 1})$ & $\mathbf{0 . 1 0}(\mathbf{0 . 6 9})$ \\
& & & & &
\end{tabular}

correction of the bias and therefore provides an additional measure to assess the performance of the bias correction scheme.

\subsection{LETKF and bias correction}

We adapt the LETKF code developed. originally by Miyoshi (2005) and Harlim and Hunt (2007) for the assimilation of rawinsonde observations to assimilate the rawinsonde and biased radiance observations on the SPEEDY model. In all these numerical experiments, we use 40 ensemble members and perform the data assimilation every $6 \mathrm{~h}$. We find that local regions containing a single vertical level and neighbourhoods of $7 \times 7$ horizontal gridpoints $(l=3)$ and one level deep $(v=0)$ provide the best analysis for rawinsonde observations alone. We find that smaller local regions of neighbourhoods containing $3 \times 3$ gridpoints $(l=1)$ and one level deep $(v=0)$ provide the best analysis when we assimilate both the rawinsonde and the simulated satellite observations. These thin local regions provide a more accurate analysis because of the coarse vertical resolution of the seven level SPEEDY model (Fertig et al., 2007). We perform numerical experiments with both the one- and two-step analysis schemes described in Section 2.3.

Ideally, the spread in the ensemble members for the forecast state and bias parameters remains large enough to realistically represent the uncertainty in the states and bias estimates (see eqs. 12-16). To avoid a collapse of the ensemble spread, the numerical experiments perform multiplicative variance inflation on the background ensemble members (both for the model and the bias variables e.g. Anderson, 2001). Furthermore, the inflation serves as a forgetting factor so that the bias parameters can account for slowly evolving biases even though persistence (eq. 8) is used for their forecast model (Hunt et al., 2007). We inflate the background covariance matrices by a factor of $\rho$ by scaling the background perturbations by $\sqrt{1+\rho}$. Each background ensemble member is then replaced by the sum of the background mean and the corresponding scaled perturbation from that mean. As will be further described in Section 4.3, in the one-step analysis scheme, the model variables are always inflated by $\rho=6 \%$, whereas the bias variables are inflated by either $\rho=6 \%$ or $\rho=$ $12 \%$. We use the same inflation factors for the first stage of the two-step analysis scheme, but inflate the background model state variables by $\rho=5 \%$ in the second stage of this implementation.

We verify the results of the LETKF analysis by computing the rms distance between the analysis and truth (rms error). Similarly, we verify the bias correction parameters by applying the ensemble mean of the estimated bias parameters to the true values of the predictors to correct the set of biased satellite observations with no noise. We refer to the rms error of these corrected satellite observations relative to the unbiased satellite observations as the observation rms error. The time averaged results are shown for the last month of the assimilation experiments (February 1982) to exclude the spin-up period.

In this study, we subtract the mean over time and space from each of the non-constant predictors before using them for the online bias correction. Thus, the bias estimated from the constant predictor $\left(p_{1}\right)$ describes the overall mean bias and the bias 
estimated from the other predictors represents variations from that mean. We create the initial bias ensemble by randomly sampling from a Gaussian distribution with mean zero and a standard deviation based on the desired initial spread of the ensemble. We find that the analysis and estimated bias parameters can be sensitive to the initial spread in the bias parameters. Specifically, we find that the bias parameters took longer to converge when their initial spread was large. The convergence is slower for experiments using three or more predictors (results not shown). However, the spread must start out large enough for the ensemble to capture the bias uncertainty. We therefore tune the initial spread in the bias parameters to minimize the analysis errors and rms of the observation error over the entire two-month period. Based on this tuning, we choose the initial standard deviation of the bias parameters to be $1 \mathrm{~K}$ for $\beta_{1}$, standard deviation $0.02 \mathrm{~K}$ $\mathrm{m}^{-1}$ for $\beta_{2}$ and $\beta_{3}$, and standard deviation $0.004 \mathrm{~K} \mathrm{~K} \mathrm{~K}^{-1}$ for $\beta_{4}$ and $\beta_{5}$. We expect that if a reasonable initial spread is used, the initial choice of the bias parameters has a negligible impact on the later estimates of the bias parameters.

\section{Results}

To verify that the magnitude of the satellite observation bias created in the form of Watts and McNally (2004) is not negligible compared with the observational error, we plot the true bias (as defined in Section 3.3) of the simulated observations at the first analysis time (06UTC 1 January 1982) in Fig. 1 for the channel corresponding to $696.052 \mathrm{~cm}^{-1}$ (panel a) and the channel corresponding to $723.029 \mathrm{~cm}^{-1}$ (panel b). We observe that the magnitude of the bias is reasonably large and locationdependent. In particular, the bias has the smallest magnitude near the poles and its magnitude increases near the tropics to almost $1 \mathrm{~K}$. Though Fig. 1 shows the bias for a particular time and particular channels, it qualitatively represents the typical structure of the simulated bias at all times and for many of the simulated channels.

The true satellite bias can be used to infer which predictors we can expect to correct for the bias most efficiently. Though the correlations between the predictors and the true bias cannot be computed in reality, they enable us to further evaluate the performance of the bias correction scheme. We plot the correlation between each of the predictors described in Section 2 and the true bias of the simulated satellite observations in Fig. 2. We observe that the true bias is most strongly correlated to predictors $p_{2}$ and $p_{3}$ for all satellite channels at a wavenumber below 712.739 $\mathrm{cm}^{-1}$. On the other hand, predictors $p_{4}$ and $p_{5}$ are more strongly correlated to the bias for wavenumbers above $715.941 \mathrm{~cm}^{-1}$. However, none of the predictors are well correlated to the bias for channels at wavenumbers 700.218 and $701.618 \mathrm{~cm}^{-1}$. This poor correlation is observed because the bias of these channels is substantially smaller than the others (a fact which we will explore later in this section). Overall, predictors $p_{3}$ and $p_{4}$ show the strongest correlations to the true bias. We also find these

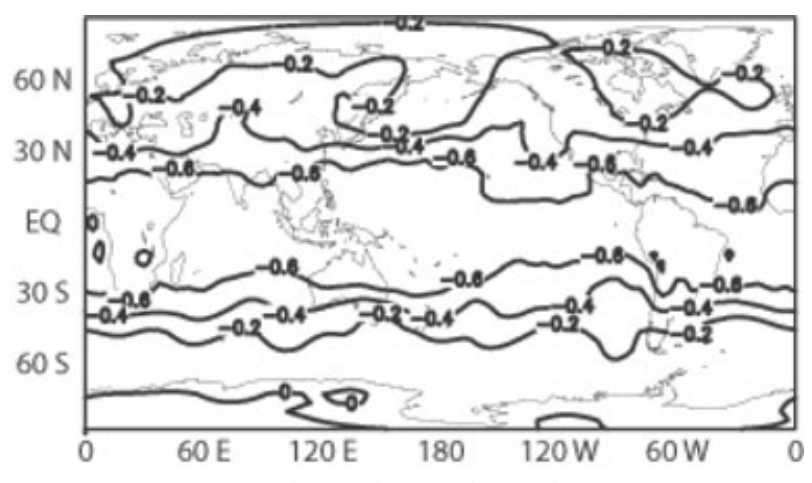

(a) Channel $696.052 \mathrm{~cm}^{-1}$

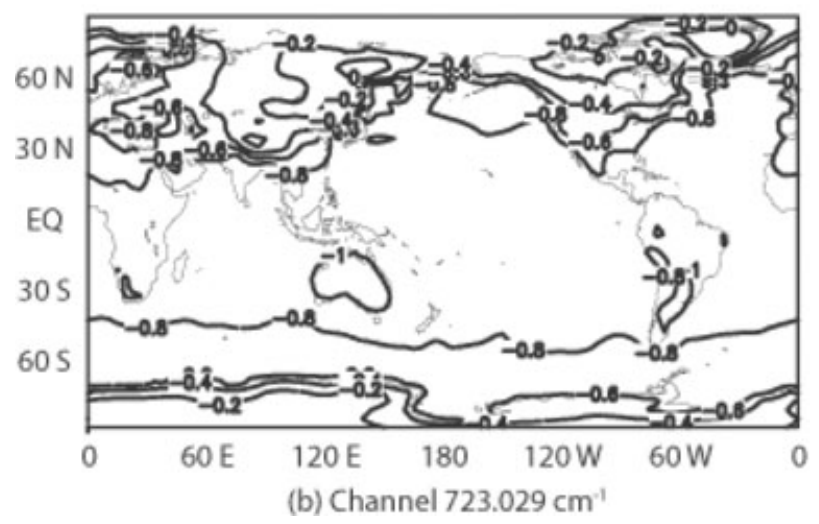

Fig 1. The true bias for two representative channels. Channel $696.052 \mathrm{~cm}^{-1}$ is in panel (a) whereas channel $723.029 \mathrm{~cm}^{-1}$ is in panel (b). The values indicated on the plot are in Kelvin.

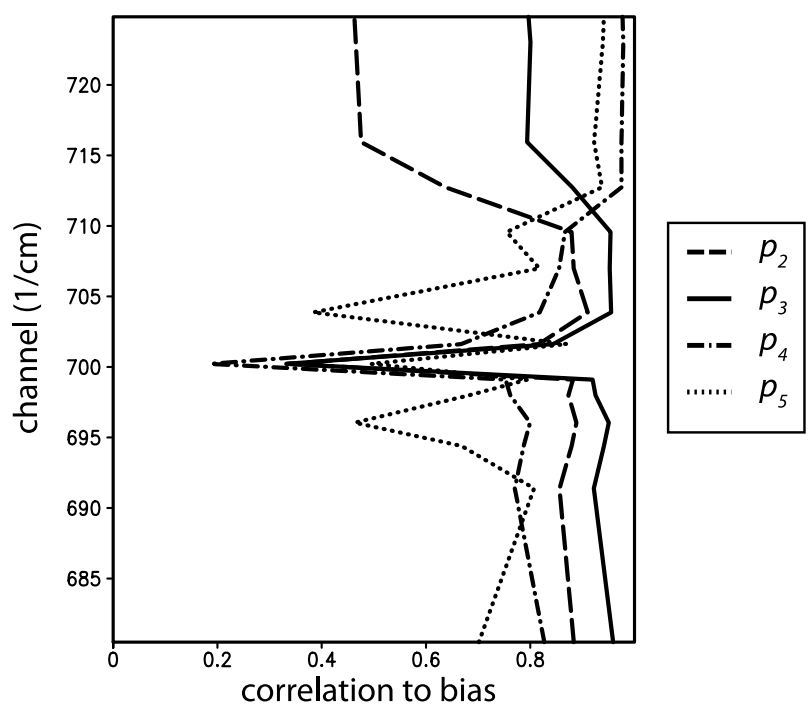

Fig 2. The correlation between the true bias and each of the predictors for each satellite channel ( $p_{2}$ is dashed; $p_{3}$ is solid; $p_{4}$ is dash-dotted and $p_{5}$ is dotted). 
Fig 3. The $500 \mathrm{hPa}$ temperature analysis rms error for each of the analysis times. The results for rawinsonde observations alone are grey and dashed, for rawinsonde and biased satellite observations using the unmodified pCRTM are grey and solid; for rawinsonde and biased satellite observations using the modified pCRTM are black, for the one-step analysis scheme are dashed, for the two-step analysis scheme are dash-dotted. Here, both analysis schemes use predictors $p_{1}, p_{3}$ and $p_{4}$ with $6 \%$ inflation for model variables and $12 \%$ inflation for bias parameters.

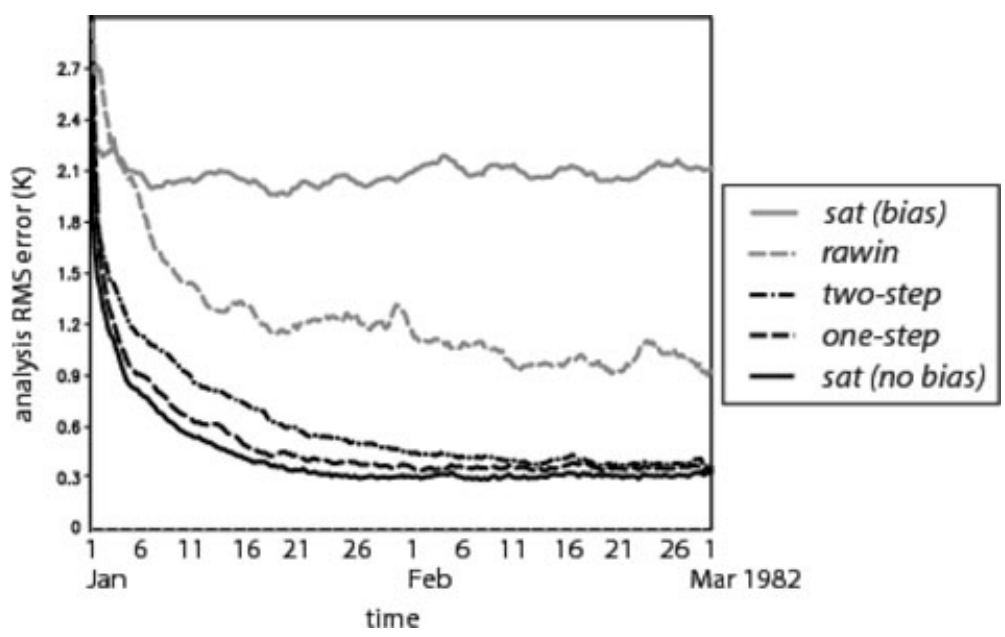

predictors to be more useful than $p_{2}$ and $p_{5}$ in reducing the analysis error in both analysis schemes. For completeness, we will show results using all five predictors at the end of this section. Here, we discuss results using only predictors $p_{1}$ (constant), $p_{3}$ (850-300 hPa thickness) and $p_{4}$ (skin temperature).

\subsection{One- and two-step analysis schemes}

In Fig. 3, we show the $500 \mathrm{hPa}$ temperature analysis rms error versus time for the one- and two-step analysis schemes with the optimal inflation factors (i.e. $6 \%$ for the model state variables and $12 \%$ for the bias variables). For reference, we plot the analysis rms error obtained when assimilating rawinsonde observations alone, rawinsonde and biased satellite observations using the unmodified pCRTM (i.e. results from satellite assimilation without bias correction) and rawinsonde and biased satellite observations using the modified pCRTM (i.e. results from satellite assimilation with the optimal observation operator). From these reference runs, it is apparent that the biased satellite observations have a significant negative impact on the analysis. Nonetheless, were these observations unbiased, they could dramatically improve the analysis over that obtained from assimilating the rawinsonde observations alone.

Both the one- and two-step analysis schemes significantly reduce the analysis rms error obtained from assimilating the simulated biased satellite radiances. The rms error of the temperature analysis at $500 \mathrm{hPa}$ reaches comparable accuracy for the two schemes, but the convergence for the two-step analysis scheme is slower than that of the one-step analysis scheme (Fig. 3). We note that we observe similar reductions in the analysis rms error for the other model variables (e.g. surface pressure and winds). However, we do not observe this more rapid convergence for variables that are not directly related to the radiance observation, for example, for the two components of the wind (results not shown).

We also explore the impact of the bias correction on the global, time averaged temperature analysis rms error for all

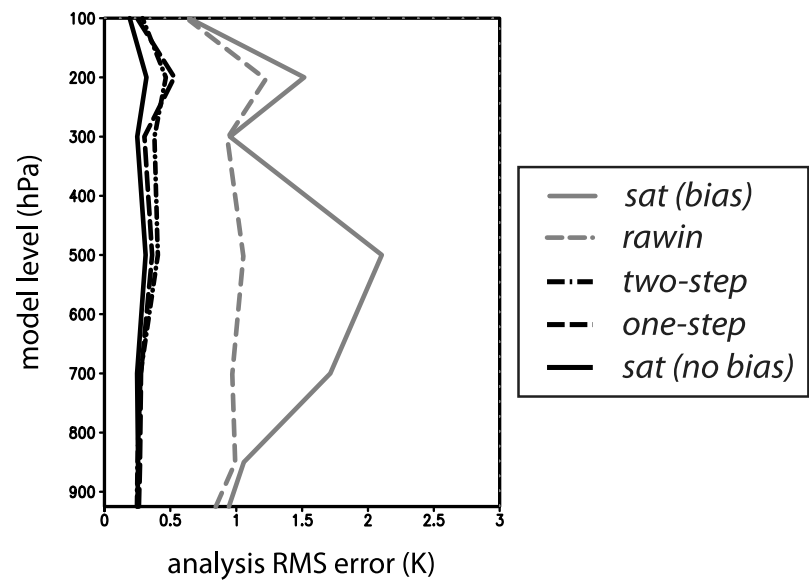

Fig 4. The global, February average of the temperature analysis rms error at each model level. The line scheme for this plot is the same as that described in Fig. 3.

seven SPEEDY model levels for both analysis schemes in Fig. 4. Both analysis schemes improve the analysis state of model state variables obtained from when assimilating the biased satellite observations with LETKF. Below $500 \mathrm{hPa}$, the analysis error from both schemes is almost as low as that obtained from assimilating the satellite observations with the true observation operator. However, the analysis error of the one-step analysis scheme is slightly smaller than that obtained from the two-step analysis scheme, reflecting its faster convergence rate.

Ideally, the bias parameters estimated by the data assimilation reduce the analysis rms errors because they reduce the bias in the observations. Figure 5 plots the global, time averaged observation rms error of all satellite channels for each analysis schemes. As in Fig. 4, we observe that the one-step scheme yields observations with slightly lower rms errors than the two-step scheme. Nonetheless, either analysis scheme significantly decreases the bias in the satellite radiances for most satellite channels. Neither bias correction scheme is able to correct the already small biases 


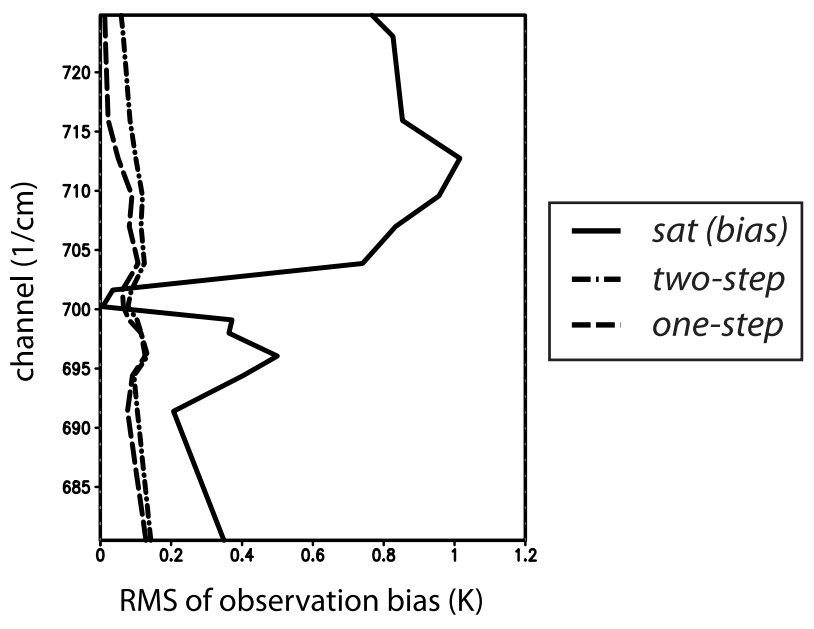

Fig 5. The global, February average of the observation rms error for each satellite channel. The observation rms error obtained from uncorrected observations without bias correction is solid; for the one-step analysis scheme are dashed; and the two-step analysis scheme are dash-dotted.

for channels at wave of 700.218 and $701.618 \mathrm{~cm}^{-1}$. Closer inspection reveals that the weighting function for these channels is such that they rarely meet the localization criterion necessary for them to be used in the analysis. Therefore, these observations have little impact on the analysis of the atmospheric variables or the bias parameters. Accordingly, the estimated bias parameters do not improve the relatively small biases for these channels.

Because the one-step analysis scheme converges faster than the two-step analysis scheme, we show results from experiments with the one-step analysis scheme in the remainder of this section. We note that we observed qualitatively similar results when conducting similar experiments with the two-step analysis scheme (results not shown).

\subsection{Observation bias-correction performance with individual bias predictors}

Although the focus of this paper is not on the choice of the predictors, examining the performance of the bias correction scheme can reveal the effects of the predictors on performance of the bias correction algorithm. In this section, we therefore compare the impact of individual predictors using the one-step analysis scheme with optimal inflation factors (6\% for model state variables and $12 \%$ for observation bias parameters). In Fig. 6, we plot the corresponding global average of the $500 \mathrm{hPa}$ temperature analysis rms error over time, obtained when simultaneously estimating bias parameters for the following combination of predictors: $p_{1}$ (dashed); $p_{1}$ and $p_{3}$ (dotted); $p_{1}$ and $p_{4}$ (dash-dotted) and $p_{1}, p_{3}$, and $p_{4}$ (dash-double dotted). All these bias correction experiments reduce the analysis rms error significantly. The skin temperature, $p_{4}$, has the most significant impact on the $500 \mathrm{hPa}$ analysis. When using $p_{1}, p_{3}$, and $p_{4}$, the $500 \mathrm{hPa}$ temperature analysis rms error more slowly tends to the value obtained from using $p_{1}$ and $p_{4}$ alone. The analysis rms error for the surface pressure is similar to the $500 \mathrm{hPa}$ temperature (results not shown). The bias correction clearly has an impact on the $500 \mathrm{hPa}$ zonal wind, meridional wind and humidity analyses (results not shown). However, there appears to be a less significant difference between the different predictors for these variables.

We plot the global, time averaged temperature analysis rms error for these one-step experiments at all seven SPEEDY model levels in Fig. 7. In this figure, we see that the $p_{4}$ reduces the analysis error most significantly for low levels, below $400 \mathrm{hPa}$. On the other hand, $p_{3}$ reduces the analysis error most significantly above $300 \mathrm{hPa}$. The combination of predictors $p_{1}, p_{3}$ and $p_{4}$ provides the analysis with the smallest error at almost all levels. We further summarize the global, time-averaged temperature rms error for all the predictors proposed in Section 2 in Table 2. This

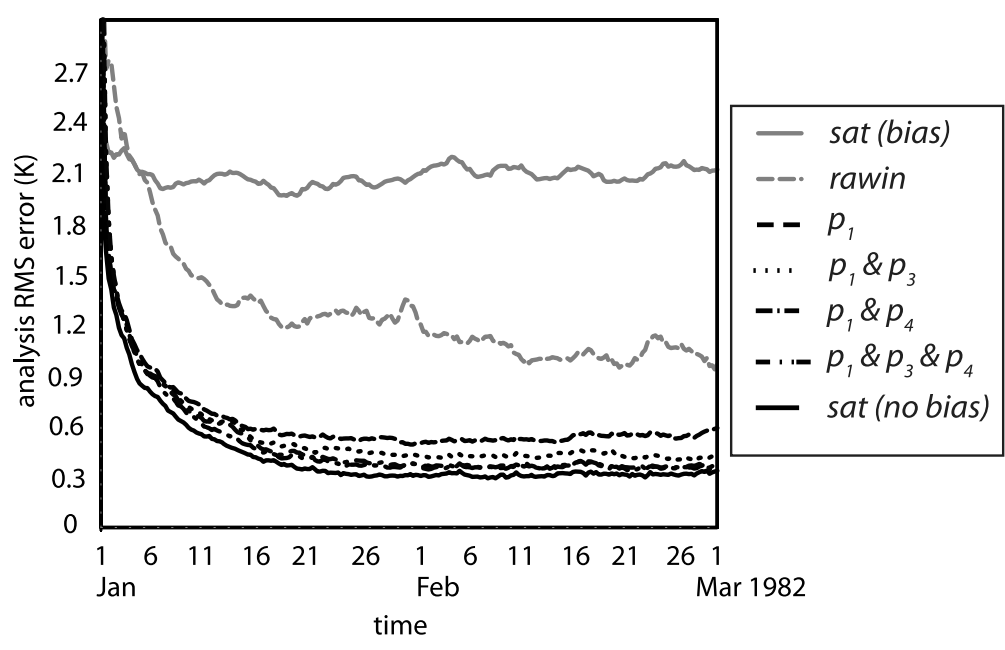

Fig 6. The $500 \mathrm{hPa}$ temperature analysis rms error for each of the analysis times using the one-step analysis scheme. The results for rawinsonde observations alone are grey and dashed, for rawinsonde and biased satellite observations using the unmodified pCRTM are grey, for rawinsonde and biased satellite observations using the modified pCRTM are black, for predictor $p_{1}$ are dashed; for predictors $p_{1}$ and $p_{3}$ are dotted and for predictors $p_{1}$ and $p_{4}$ are dash-dotted and $p_{1}$, $p_{3}$ and $p_{4}$ are dash-double-dotted. Here, the model variables are inflated by $6 \%$ and the bias parameters by $12 \%$. 


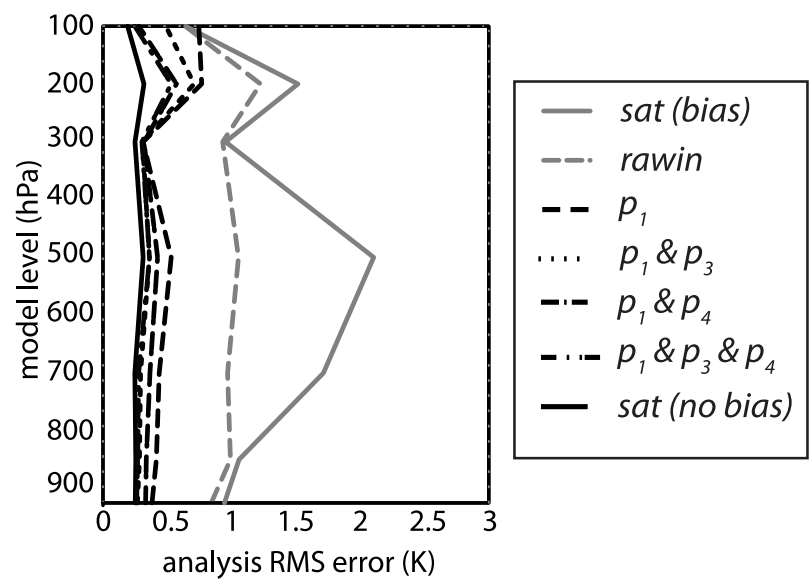

Fig 7. The global, February average of the temperature analysis rms error at each model level using the one-step analysis scheme. The line scheme for this plot is the same as that used in Fig. 6.

Table 2. The global, February average of the temperature analysis rms error $(\mathrm{K})$ at each model level for selected combinations of predictors. Here, $6 \%$ variance inflation is applied to the model variables and $12 \%$ variance inflation is applied to the bias parameters

\begin{tabular}{lccccccc}
\hline & \multicolumn{7}{c}{ Model level } \\
\cline { 2 - 8 } Experiment & 1 & 2 & 3 & 4 & 5 & 6 & 7 \\
\hline $\begin{array}{l}\text { Modified pCRTM } \\
\text { (unbiased) }\end{array}$ & 0.25 & 0.25 & 0.25 & 0.31 & 0.25 & 0.32 & 0.19 \\
$p_{1}$ & & & & & & & \\
$p_{1}, p_{2}$ & 0.38 & 0.41 & 0.44 & 0.53 & 0.31 & 0.77 & 0.75 \\
$p_{1}, p_{3}$ & 0.37 & 0.40 & 0.44 & 0.51 & 0.34 & 0.60 & 0.33 \\
$p_{1}, p_{4}$ & 0.33 & 0.34 & 0.37 & 0.42 & 0.32 & 0.57 & 0.28 \\
$p_{1}, p_{5}$ & 0.27 & 0.28 & 0.29 & 0.36 & 0.31 & 0.71 & 0.49 \\
$p_{1}, p_{3}, p_{4}$ & 0.29 & 0.30 & 0.32 & 0.38 & 0.32 & 0.77 & 0.60 \\
$p_{1}, p_{2}, p_{3}, p_{4}, p_{5}$ & 0.26 & 0.27 & 0.28 & 0.36 & 0.30 & 0.53 & 0.25 \\
\hline & 0.26 & 0.27 & 0.28 & 0.39 & 0.33 & 0.56 & 0.34 \\
\hline
\end{tabular}

table shows that using $p_{2}$ or $p_{5}$ also decreases the analysis rms error but not as significantly as using $p_{3}$ or $p_{4}$. Also, including $p_{2}$ and $p_{5}$ in the bias correction with $p_{1}, p_{3}$ and $p_{4}$ does not significantly reduce the analysis rms error.

Figure 8 plots the global, time averaged observation rms error for each of the choices of predictors for each satellite channel. We observe that $p_{3}$ decreases the rms of the observation error most for low wavenumbers, whereas $p_{4}$ decreases the rms of the observation error for higher wavenumbers. This result is consistent with the structure of the correlation between the true observation bias and these predictors shown in Fig. 2. Furthermore, using predictors $p_{1}, p_{3}$ and $p_{4}$ together significantly reduces the observation rms for most channels. The global, time averaged observation rms error is summarized for selected channels using all choices of predictors in Table 3. As for the analysis errors, we see that none of the results using $p_{2}$ or $p_{5}$ improve significantly compared with the results using $p_{3}$ and/or $p_{4}$. We also see that

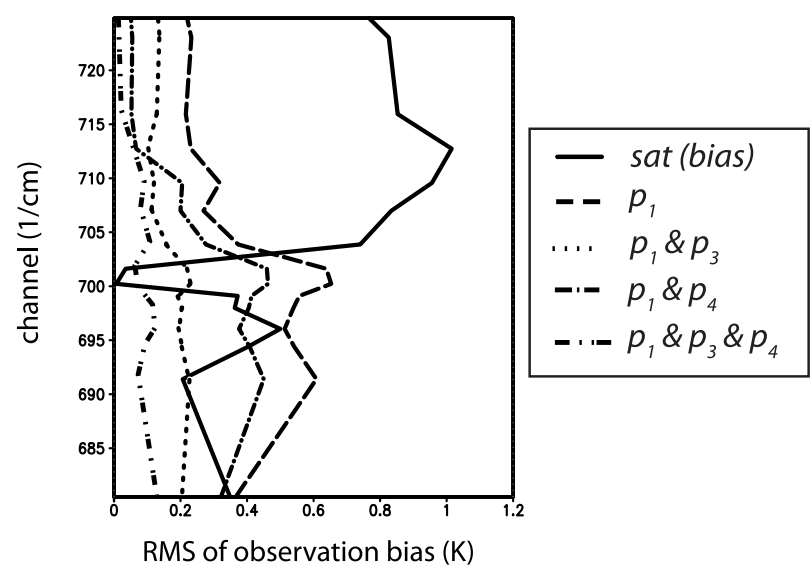

Fig 8. The global, February average of the observation rms error for each satellite channel using the one-step analysis scheme. The observation rms error obtained when without bias correction is solid. The results using bias correction with predictor $p_{1}$ is dashed, $p_{1}$ and $p_{3}$ is dotted, for $p_{1}$ and $p_{4}$ is dash-dotted and $p_{1}, p_{3}$ and $p_{4}$ is dash-double-dotted.

Table 3. The global, February average of the observation rms error $(\mathrm{K})$ for representative channels when applying the bias correction parameters estimated using $6 \%$ variance inflation for the model variables and $12 \%$ for the bias parameters

\begin{tabular}{lcccc}
\hline & \multicolumn{4}{c}{ Channel $\left(\mathrm{cm}^{-1}\right)$} \\
\cline { 2 - 5 } Experiment & 680.491 & 696.052 & 709.566 & 723.029 \\
\hline Unmodified pCRTM & 0.35 & 0.50 & 0.96 & 0.83 \\
(biased) & & & & \\
$p_{1}$ & 0.37 & 0.51 & 0.32 & 0.23 \\
$p_{1}, p_{2}$ & 0.20 & 0.21 & 0.16 & 0.21 \\
$p_{1}, p_{3}$ & 0.21 & 0.20 & 0.12 & 0.14 \\
$p_{1}, p_{4}$ & 0.32 & 0.38 & 0.20 & 0.05 \\
$p_{1}, p_{5}$ & 0.37 & 0.47 & 0.27 & 0.09 \\
$p_{1}, p_{3}, p_{4}$ & 0.13 & 0.13 & 0.09 & 0.01 \\
$p_{1}, p_{2}, p_{3}, p_{4}, p_{5}$ & 0.19 & 0.12 & 0.14 & 0.02 \\
\hline
\end{tabular}

for each of the channels, the predictors that are best correlated with the true bias (see Fig. 2) yield the greatest improvement in the observation rms error.

We observe in Fig. 8 that for the lower wavenumber channels, using certain combinations of the predictors increases the rms error of the observations above their value with no bias correction. However, the bias correction still significantly improves the analysis rms error throughout the atmosphere (Fig. 7). For example, whereas using $p_{1}$ (constant) improves the analysis at all model levels but the highest, this predictor only improves the observation rms error for channels with wavenumbers above $701.618 \mathrm{~cm}^{-1}$ (Fig. 8). To explore the impact of the increased observation rms error further, we tried using $p_{1}$ to correct only the bias for channels above $701.618 \mathrm{~cm}^{-1}$. We plot the resulting 


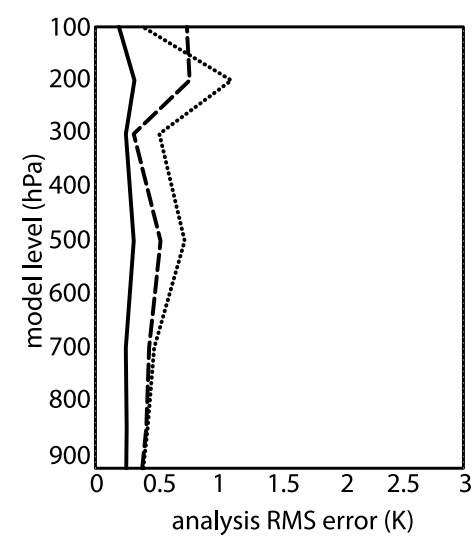

(a) Temperature Analysis RMS Error

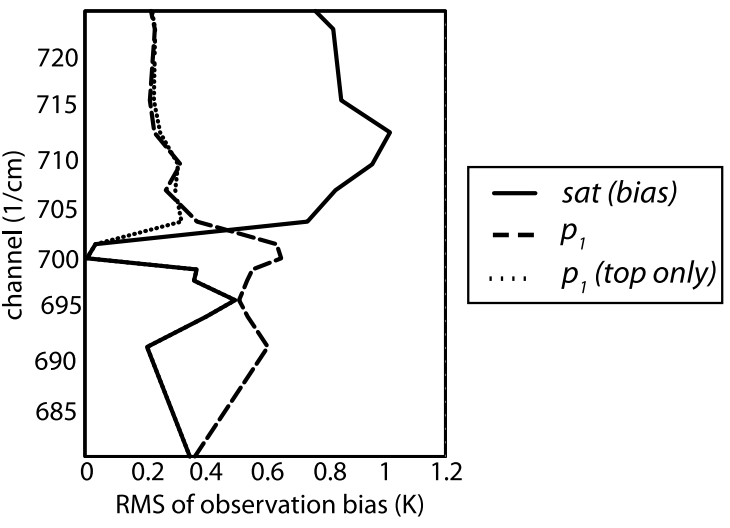

(b) Observation RMS Error

Fig 9. The results from using $p_{1}$ to correct only channels above $701.618 \mathrm{~cm}^{-1}$ (dotted) compared eith using $p_{1}$ at all levels (dashed). In panel (a) showing the temperature analysis, we plot the results obtained from using the true modified observation operator (solid). In panel (b) showing the rms error of the observations, the true bias is plotted (solid) for reference. All experiments here use the one-step analysis scheme.

observation and analysis rms errors in panels (a) and (b) of Fig. 9, respectively. The observation rms error from using $p_{1}$ to correct the bias only for channels above wavenumber $701.618 \mathrm{~cm}^{-1}$ (dotted) mirrors that obtained from using $p_{1}$ for all channels (dashed) above $701.618 \mathrm{~cm}^{-1}$ and the true rms of the observation error (solid in panel (a)) for the rest of the channels. In spite of this apparent improvement in the overall observation rms error, using $p_{1}$ to correct the bias only for channels above $701.618 \mathrm{~cm}^{-1}$ degrades the temperature analysis rms error for the six lower model levels.

\subsection{Inflation of model state variables and bias parameters}

In the experiments presented in the previous sections, we have used different inflation factors for the model state variables and bias parameters. However, as formulated in Hunt et al. (2007), LETKF applies a single inflation factor to all variables in the ensemble. In this section, we illustrate the need for different inflation factors by comparing results from numerical experiments using a $6 \%$ inflation factor for all variables to those described above.

Table 4 shows the global, time averaged temperature analysis rms error at all model levels using 6\% variance inflation. We observe that the analysis rms errors obtained here are similar in magnitude to those obtained by employing two different inflation factors (summarized in Table 2). For the online bias correction to continually update the bias estimate, the spread (standard deviation) of the ensemble of bias parameters must remain significantly larger than zero. Therefore, Fig. 10 plots the background spread of each of the bias parameters for a representative subset of the channels corrected in the run with $p_{1}$, $p_{3}$ and $p_{4}$ (panels a, $\mathrm{b}$ and $\mathrm{c}$, respectively) over time. The spread in the bias parameters for channel $723.029 \mathrm{~cm}^{-1}$ remains nearly
Table 4. As for Table 2. Here, $6 \%$ variance inflation is applied to both the model variables and bias parameters

\begin{tabular}{lccccccc}
\hline & \multicolumn{7}{c}{ Model level } \\
\cline { 2 - 8 } Experiment & 1 & 2 & 3 & 4 & 5 & 6 & 7 \\
\hline $\begin{array}{l}\text { Modified pCRTM } \\
\text { (unbiased) }\end{array}$ & 0.25 & 0.25 & 0.25 & 0.31 & 0.25 & 0.32 & 0.19 \\
$p_{1}$ & 0.40 & 0.44 & 0.46 & 0.53 & 0.30 & 0.79 & 0.79 \\
$p_{1}, p_{3}$ & 0.33 & 0.34 & 0.37 & 0.42 & 0.28 & 0.46 & 0.25 \\
$p_{1}, p_{4}$ & 0.27 & 0.28 & 0.28 & 0.35 & 0.29 & 0.72 & 0.58 \\
$p_{1}, p_{3}, p_{4}$ & 0.29 & 0.29 & 0.31 & 0.38 & 0.30 & 0.50 & 0.28 \\
\hline
\end{tabular}

constant after the spin-up time. However, for the majority of the other channels the spread of the bias parameters decreases dramatically as the analysis cycle proceeds. A similar decrease in the bias spread is observed for bias correction experiments with all combinations of the predictors (results not shown). For channels like $723.029 \mathrm{~cm}^{-1}$, where the bias remains nearly constant, we observe that the weighting function is above the cut-off value for several model levels (see Table 1). Therefore, the analysis of the bias parameters corresponding to these channels combines information from more local regions, keeping the spread relatively large.

A sufficiently large multiplicative variance inflation factor can prevent the background ensemble from collapsing and also serves as a forgetting factor of past observations and dynamics. However, increasing the variance inflation of all background variables to a level that prevents the ensemble of bias parameters from collapsing degrades the quality of the analysis (results not shown). Thus, we compensate for the decreasing spread in the ensemble of bias parameters by inflating these variables by $12 \%$ and retaining the $6 \%$ variance inflation for the other 


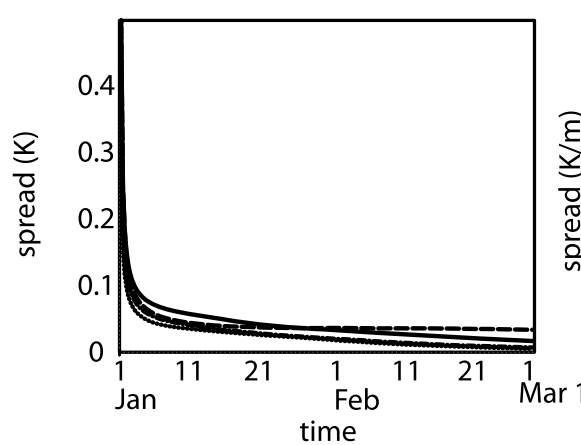

(a) spread of $\beta_{1}$

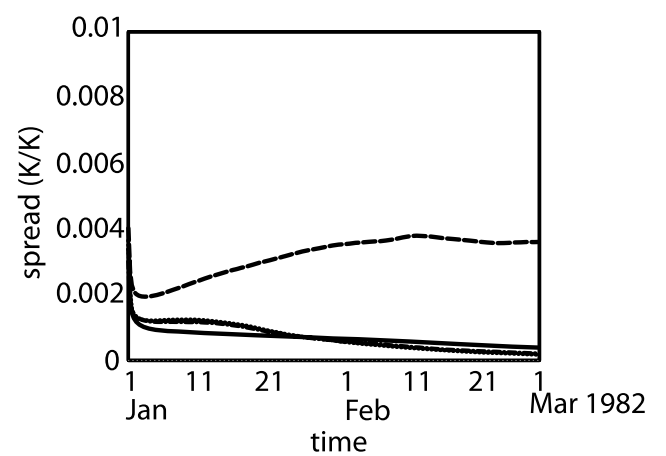

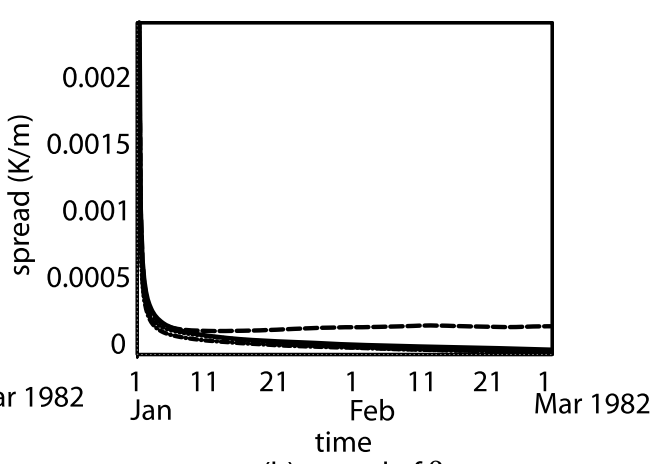

(b) spread of $\beta_{3}$

(c) spread of $\beta_{4}$

Fig 10. The spread in the background bias parameters $\beta_{1}, \beta_{2}$ and $\beta_{4}$ for the run with predictors $p_{1}, p_{3}$ and $p_{4}$ for the one-step analysis scheme. The spread of these parameters is in panels (a), (b) and (c), respectively. Each line represents a different satellite channel, where the solid line is for channel $680.491 \mathrm{~cm}^{-1}$, the dotted line is for channel $696.052 \mathrm{~cm}^{-1}$, the dash-dotted line is for channel $709.566 \mathrm{~cm}^{-1}$ and the dashed line is for channel $723.029 \mathrm{~cm}^{-1}$.

analysis variables. As described above, the analysis rms error is similar when using a universal inflation factor or different inflation factors. However, Fig. 11 shows that the spread of the bias parameters for the run with $p_{1}, p_{3}$ and $p_{4}$ (panels $\mathrm{a}, \mathrm{b}$ and (c), respectively) appears to level off toward a non-zero value for most channels. The results are similar for the other bias correction experiments (results not shown).

\section{Conclusions}

In this paper we have presented and tested two localized ensemble Kalman filter based schemes designed to correct for observational bias in satellite radiance data. We refer to these as the 'one-step analysis scheme' and 'two-step analysis scheme'. In perfect model experiments with simulated rawinsonde and biased satellite radiance observations, both schemes yield analyses with substantially smaller errors than those obtained without bias correction and those obtained from assimilating only rawinsonde observations. Furthermore, even though the form of the bias parametrization in our analysis differs from the form of the simulated bias in our observations, after a relaxation period, both schemes perform nearly as well as does the analysis obtained from assimilating unbiased satellite observations. Even though the analysis rms error always decreases, we sometimes observe an increase in the observation rms errors due to the bias correction. We speculate that this behaviour occurs because the adaptive bias correction schemes presented in this paper seek bias parameters that will minimize the analysis rms error.

Our two bias correction schemes differ in how they resolve the conflict posed by the practical computational necessity of localization versus the fact that the instrument bias parameters are global (i.e. not spatially dependent). In either case, this conflict necessitates giving up something. In our one-step method, we retain background correlations between the bias parameters and the state variables but introduce the possibility of local overfitting by the bias parameters. In our two-step method, we remove this possibility but in doing so, also remove correlations between the bias parameters and the atmospheric state variables in updates of the atmospheric state variables. Our results show that both methods yield about the same accuracy after enough time is allowed for relaxation, but the relaxation time for the one-step method is substantially shorter. Since the two methods yield comparable accuracy and are not much less accurate than the analysis with unbiased observations, we speculate that local overfitting may not be a significant problem in our application. We believe that this is because the ensemble provides a strong constraint ensuring that the local analyses only make a small change from the globally constant forecasted bias parameter. Since the one-step 


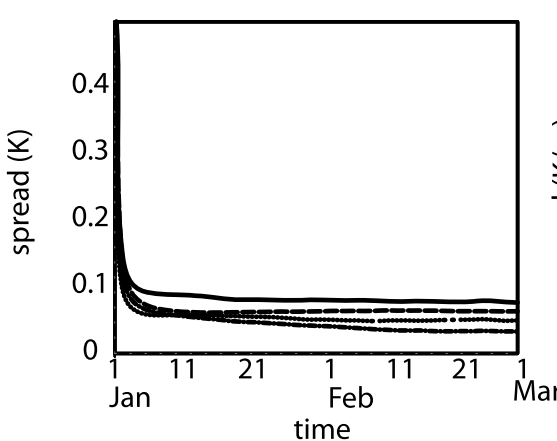

(a) spread of $\beta_{1}$

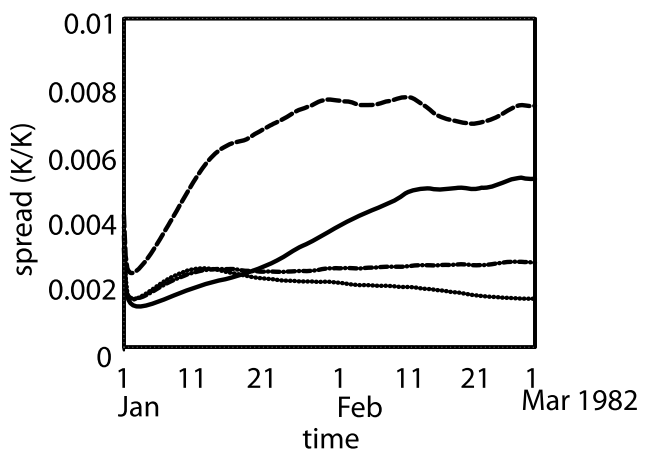

(c) spread of $\beta_{4}$

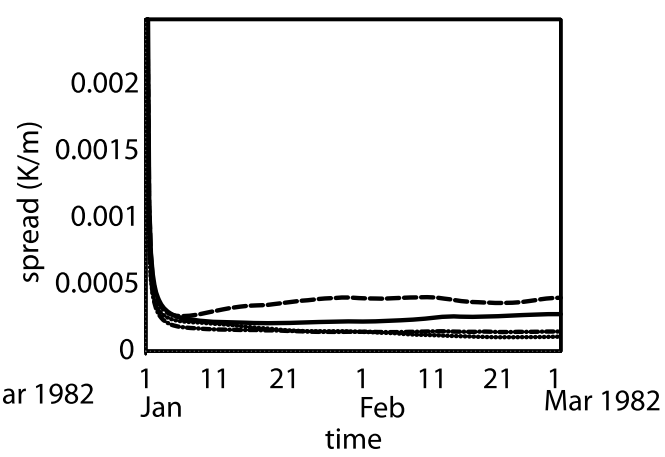

(b) spread of $\beta_{3}$

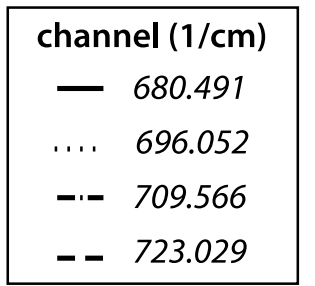

Fig 11. As for Fig. 10. Here, the analysis variables are inflated by $6 \%$ whereas the bias parameters are inflated by $12 \%$.

method relaxes to its good result more quickly, we speculate that inclusion of correlations between the atmospheric state variables and bias parameters speeds relaxation. The faster relaxation of the one-step method should allow it to more readily adjust to temporal drifts of the bias parameters.

The overall quite positive impact of our bias correction schemes in the tests we have carried out suggests that these methods may be useful in practice. An indication that this may be the case is provided by our preliminary work presented in Szunyogh et al. (2007). In that study, we used an implementation of the one-step analysis scheme with LETKF on the model component of the NCEP GFS to assimilate advanced microwave sounding unit instrument A (AMSU-A) radiance observations from the satellite Aqua. Adding the AMSU-A radiance observations to the assimilated data set led to a major improvement of the temperature and wind forecasts in the Southern Hemisphere.

In assimilations of observations of the real atmosphere, model errors are necessarily combined with observation biases. The preliminary results presented in Szunyogh et al. (2007) suggest that the bias correction scheme presented here performs well for real observations, although in practice, it cannot distinguish between these two sources of error. In theory, the one-step scheme may overfit the model biases in local regions. However, as discussed above, we expect that the strong constraint of the background ensemble and global averaging would mitigate this effect. Using the two-step analysis scheme would also reduce the introduction of extra degrees of freedom. Furthermore, this two-step analysis scheme could be modified to incorporate ad- ditional past observations, as described in Auligne et al. (2007) to reduce the introduction of model biases into the estimates of observation bias parameters. We are continuing to explore this mixture of model and observation biases in future studies with observations of the real atmosphere.

\section{Acknowledgments}

The authors thank Takemasa Miyoshi for adapting the SPEEDY model for data assimilation and Takemasa Miyoshi and John Harlim for developing the original LETKF code for the SPEEDY model. The authors also thank Matt Cornick, Jim Jung, Ricardo Todling, Jeffrey Whitaker and the reviewers for their helpful suggestions. This work was supported by NOAA/THORPEX (Grant NA04OAR4310104), NSF (Grants ATM034225 and ATM0722721) and NASA (Grants NNG004GK29G, NNX08AD40G and NNX07AV45G). E.J.F. would also like to acknowledge the support of University of Maryland's Earth System Science Interdisciplinary Center and NASA Goddard Space Flight Center.

\section{References}

Anderson, J. L. 2001. An ensemble adjustment Kalman filter for data assimilation. Mon. Wea. Rev. 129, 2884-2903.

Auligne, T., McNally, A. P. and Dee, D. P. 2007. Adaptive bias correction for satellite data in a numerical weather prediction system. Quart. J. R. Meteorol. Soc. 133, 631-642. 
Baek, S.-J., Hunt, B. R., Kalnay, E., Ott, E. and Szunyogh, I. 2006. Local ensemble Kalman filtering in the presence of model bias. Tellus $\mathbf{5 8 A}$, 293-306.

Cameron, J. R. N. 2003. The effectiveness of the AIRS bias correction for various air-mass predictor combinations. Technical Report, Met. Office NWP. Available at: http://www.metoffice.com/research/nwp/ publications/papers/technical_reports/2003/FRTR421/FRTR421.pdf.

Dee, D. P. 2005. Bias and data assimilation. Quart. J. R. Meteorol. Soc. 131, 3323-3343.

Dee, D. P. and da Silva, A. M. 1998. Data assimilation in the presence of forecast bias. Quart. J. R. Meteorol. Soc. 124, 269-295.

Derber, J. C. and Wu, W.-S. 1998. The use of TOVS cloud-cleared radiances in the NCEP SSI analysis system. Mon. Wea. Rev. 126, 2287-2299.

Eyre, J. R. 1992. A bias correction scheme for simulated TOVS brightness temperatures. Technical Memorandum 186, ECMWF, Reading, UK.

Fertig, E. J., Hunt, B. R., Ott, E. and Szunyogh, I. 2007. Assimilating nonlocal observations with a local ensemble Kalman filter. Tellus 59A, 719-730.

Friedland, B. 1969. Treatment of bias in recursive filtering. IEEE Trans. Auto. Control 14, 359-367.

Hamill, T., Whitaker, J. S. and Snyder, C. 2001. Distance-dependent filtering of background covariance estimates in an ensemble Kalman filter. Mon. Wea. Rev. 129, 2776-2790.

Han, Y., van Delst, P., Liu, Q., Weng, F. and Derber, J. C. 2005. User's Guide to the JCSDA Community Radiative Transfer Model (Beta Version). Joint Center for Satellite Data Assimilation, Camp Springs, MD, USA.

Harlim, J. and Hunt, B. R. 2007. A non-Gaussian ensemble filter for assimilating infrequent noisy observations. Tellus 59A, 225-237.

Harris, B. A. and Kelly, G. 2001. A satellite radiance-bias correction scheme for data assimilation. Quart. J. R. Meteorol. Soc. 127, 14531468.

Harris, B. A., Cameron, J., Collard, A. and Saunders, R. 2004. Effect of air-mass predictor choice on the AIRS bias correction at the Met Office. In: Proceedings of the Thirteenth International TOVS Study Conference, International ATOVES Working Group, Sainte-Adele, Quebec, Canada, 92-98.

Houtekamer, P. L. and Mitchell, H. L. 2001. A sequential ensemble Kalman filter for atmospheric data assimilation. Mon. Wea. Rev. 129, 123-137.

Houtekamer, P. L. and Mitchell, H. L. 2006. Ensemble Kalman filtering. Quart. J. R. Meteorol. Soc. 131, 3269-3289.

Houtekamer, P. L., Mitchell, H. L., Pellerin, G., Buehner, M., Spacek, L. and co-author. 2005. Atmospheric data assimilation with the ensemble Kalman filter: results with real observations. Mon. Wea. Rev. 133, 604-620.

Hunt, B. R., Kostelich, E. J. and Szunyogh, I. 2007. Efficient data assimilation for spatiotemporal chaos: a local ensemble transform Kalman filter. Physica D. 230, 112-126.

Kalnay, E. 2003. Atmospheric Modelling, Data Assimilation and Predictability. Cambridge University Press, New York, 364 pp.

Kalnay, E., Hunt, B. R., Ott, E. and Szunyogh, I. 2006. Ensemble forecasting and data assimilation: two problems with the same solution? In: Predictability of Weather and Climate (eds. T. N. Palmer and R. Hagedorn). Cambridge University Press, New York.
Keppenne, C. L., Rienecker, M. M., Kurowski, N. P. and Adamec, D. A. 2005. Ensemble Kalman filter assimilation of temperature and altimeter data with bias correction and application to seasonal prediction. Nonlinear Proc. Geophys. 12, 491-503.

Kuhl, D., Szunyogh, I., Kostelich, E. J., Patil, D. J., Gyarmati, and coauthors. 2007. Assessing predictability with a local ensemble Kalman filter. J. Atmos. Sci. 64, 1116-1140.

Le Marshall, J., Jung, J., Zapotocny, T., Derber, J., Treadon, R., and co-authors. 2006. The application of AIRS radiances in numerical weather prediction. Aust. Meteorol. Mag. 55, 213-217.

Liou, K. 2002. An Introduction to Atmopsheric Radiation 2nd Edition. Academic Press, New York, 583 pp.

Liu, J., Fertig, E. J., Li, H., Kalnay, E., Hunt, B. R., and co-authors. 2008. Comparison between local ensemble transform Kalman filter and PSAS in the NASA finite volume GCM perfect model experiments. Nonlinear Proc. Geophys. 15, 645-659.

Miyoshi, T. 2005. Ensemble Kalman filter experiments with a primitiveequation global model. PhD Thesis, University of Maryland, College Park, MD, USA.

Molteni, F. 2003. Atmospheric simulations using a GCM with simplified physics parameterizations, I: model climatology and variability in multi-decadal experiments. Clim. Dyn. 20, 175-191.

Oczkowski, M., Szunyogh, I. and Patil, D. J. 2005. Mechanisms for the development of locally low dimensional atmospheric dynamics. J. Atmos. Sci. 62, 1135-1156.

Ogata, K. 1990. Modern Control Engineering 2nd Edition. Prentice Hall, Englewood Cliffs, NJ, 960 pp.

Ott, E., Hunt, B. R., Szunyogh, I., Zimin, A., Kostelich, E. J., and coauthors. 2004. A local ensemble Kalman filter for atmospheric data assimilation. Tellus 56A, 415-428.

Patil, D. J., Hunt, B. R., Kalnay, E., Yorke, J. A. and Ott, E. 2005. Local low dimensionality of atmospheric dynamics. Phys. Rev. Lett. 86, 5878-5881.

Rizzi, R. and Matricardi, M. 1998. The use of TOVS clear radiances for numerical weather prediction using an updated forward model. Quart. J. R. Meteorol. Soc. 124, 1293-1312.

Susskind, J., Barnet, C. and Blaisdell, J. 2003. Retrieval of atmospheric and surface parameters from AIRS/AMSU/HSB under cloudy conditions. IEEE Trans. Geosci. Remote Sens. 41, 390-409.

Szunyogh, I., Kostelich, E. J., Gyarmati, G., Patil, D. J., Kalnay, E., and co-authors. 2005. Assessing a local ensemble Kalman filter: Perfect model experiments with the National Centers for Environmental Prediction global model. Tellus 57A, 528-545.

Szunyogh, I., Satterfield, E. A., Aravequia, J. A., Fertig, E. J., Gyarmati, G., and co-authors. 2007. The local ensemble transform Kalman filter and its implementation on the NCEP global model at the University of Maryland. In: Proceedings of the ECMWF Workshop on Flowdependent aspects of data assimilation, ECMWF, Reading, United Kingdom, 47-64.

Szunyogh, I., Kostelich, E. J., Gyarmati, G., Kalnay, E., Hunt, B. R., and co-authors. 2008. A local ensemble transform Kalman filter data assimilation system for the NCEP global model. Tellus 60A, 113130.

Turner, D. S. 1994. HIRS sensitivity to $\mathrm{CO}_{2}$ mixing ratio and a pragmatic correction term. J. Appl. Meteorol. 33, 1155-1162.

Watts, P. D. and McNally, A. P. 2004. Identification and correction of radiative transfer modelling errors for atmospheric sounders: AIRS and 
AMSU-A. In: Proceedings of the ECMWF Workshop on Assimilation of high spectral resolution sounders in NWP, ECMWF, Reading, United Kingdom, 28 June-1 July, 2004.

Whitaker, J. S. and Hamill, T. M. 2002. Ensemble data assimilation without perturbed observations. Mon. Wea. Rev. 130, 19131924.
Whitaker, J. S., Hamill, T. M., Wei, X., Song, Y. and Toth, Z. 2008. Ensemble data assimilation with the NCEP global forecast system. Mon. Wea. Rev. 463-482.

Zupanski, D. and Zupanski, M. 2006. Model error estimation employing an ensemble data assimilation approach. Mon. Wea. Rev. 134, 13371354. 